\title{
Personal Health Book Application for Developing Countries
}

\author{
Seddiq Alabbasi, Andrew Rebeiro-Hargrave, Kunihiko Kaneko, Ashir Ahmed and Akira Fukuda \\ Department of Advanced Information Technology Kyushu University \\ Fukuoka, Japan
}

\begin{abstract}
We introduce a Personal Health Book application that is used as a portable repository for Personal Health Records (PHR) in order to alleviate healthcare organizational problems in developing countries. The Personal Health Book application allows low literate people to access and carry their own medical history from a rural healthcare provider to an urban healthcare provider. This will improve the efficiency of medical care and lower costs for health clinics in underserved areas. This paper introduces a software application that can be ported onto a USB Smart Card or/and managed by smartphone or personal computer connected to cloud computing environment. The Portable Health Book application aims to ease the problem of interoperability between health clinics by accepting any file format and contents and applies a decomposed database to categorize, group and reorganize the data. Querying the application's database, the consumer can create a unified report presentation that is understandable by the consumer, family, and healthcare provider. We tested the Personal Health Book framework by importing PHRs in an extensible markup language (XML) format with a basic structure, without checking the PHR content from the Grameen Portable Health Clinic database in Bangladesh and from different departments from a hospital in Japan. The Personal Health Book was able to generate a human readable output as its database reorganize and store any type of PHR including sensor device data.
\end{abstract}

Keywords-Personal health records; Patient centered healthcare; Database design; Developing countries; Extensible markup language

\section{INTRODUCTION}

The introduction of an affordable and small Personal Health Book software application that stores Personal Health Records can help alleviate healthcare organizations' problems in developing countries.

Personal Health Records (PHR) are a set of computer-based tools that allow people to access and coordinate their lifelong health information and make appropriate parts of it available to those who need it $[1,2]$. PHR systems were developed in the late 1990s to target patients who were travelling and needed healthcare and for situations where patients were not able to provide their health information $[3,4]$, or have communication difficulties.

Currently, three types of PHRs have become available [5]. These include: stand-alone formats (PC, USB drive), where consumers store health information on personal computers but lack the ability to exchange information between consumers and healthcare provides; Web formats that are managed by third parties (such as Microsoft Healthvalut, Dossia
Consortium) and allow consumers to maintain their health information on private online accounts accessed by a login ID and password; Integrated PHRs with Electronic Health Records (EHR) where a healthcare provider (such as MyHealthVet of the US Department of Veteran Affairs) combines patient entered content with EHR data [6].

Despite significant interest and anticipated benefits for consumers and healthcare providers, the overall adoption of PHRs remains relatively low [7]. A study on MyHealthVet PHR system showed the authentication and secure messaging had important consequences for access, communication, patient self-report and patient/provider relationship. From a literature review of 28 articles the common challenges for the use of PHRs include: data accuracy, data privacy and security, digital divide, and literacy issues.

Accepting the PHR challenges, the concept of a Personal Health Book (PHB) has been introduced to the literature. Similar to Web formats and online PHR health vaults, the Personal Health Book-based healthcare model is a cloud-based service where consumer PHRs are stored at a remote server and shared with healthcare providers (such as a pharmacy) whom the consumer authorizes and who has the capacity to import data from other information sources [8,9]. An alternative approach is to revisit the "stand-alone approach" and let the consumer have a small "personal health book" software application that stores different types of PHRs from different healthcare providers. This is similar to a diary that houses "personal lifetime data" and is an application with standard interfaces and its own database [10].

A simple digital Personal Health Book that stores PHRs can overcome the digital divide and improve the efficiency of healthcare organization in developing countries by enabling people to carry their own medical history. Currently, millions of low-income people frequently travel from rural communities to urban centres and do not have any access to their own medical records. When patients revisit health clinics, doctors often waste their time and resources finding and organizing patients' information in often chaotic, difficult to manage, paper-based systems [11]. The Personal Health Book can partially alleviate these problems by moving some of the responsibility for preservation of basic medical records from institutions to the patient.

This paper introduces the concept of Portable Health Book (PHB) for developing countries. The PHB is a patient-centred software application that enables consumers to collect health information from one health clinic, view the information, and 
take the information to another health clinic. The paper introduces a PHB use-case for offline USB Smart Card and online smart phone cloud environment storage.

In the Methodology section, we introduce a PHB usability use-case in a developing country context and simplified interoperability requirements. In the technical design description and user interface sections, we show how to import data from a source health clinic, describe the structure of an adaptable decomposed database, and indicate how the stored data can be viewed. We then test the PHB framework by importing PHRs from $\mathrm{s}$ developing country database and Japanese hospital and discuss the results and implications.

\section{MethodOLOGY}

\section{A. Portable Health Book Usability}

The implementation of a digital Portable Health Book in developing countries requires affordability (low cost) and simplicity (ease of use). Following these drivers are robustness, privacy, and security. For this study, it is suggested that a USB or Smart Card [12] is a suitable and affordable solution for the low-income person. The "ease of use" depends on the literacy level of the consumer and willingness of the healthcare provider to share the PHR. We envisage that the consumer will ask the healthcare provider to store the resultant PHR or EHR on their USB or Smart Card. This is applicable to rural community health clinics or visiting health providers such as the Grameen Portable Health Clinic in Bangladesh [13]. At the rural community, health clinics store computer records and give patients printed prescriptions. It is feasible to ask for a digital copy. Once imported a USB or Smart Card, the consumer will store the PHR and then can view PHB data using a PC or tablet.

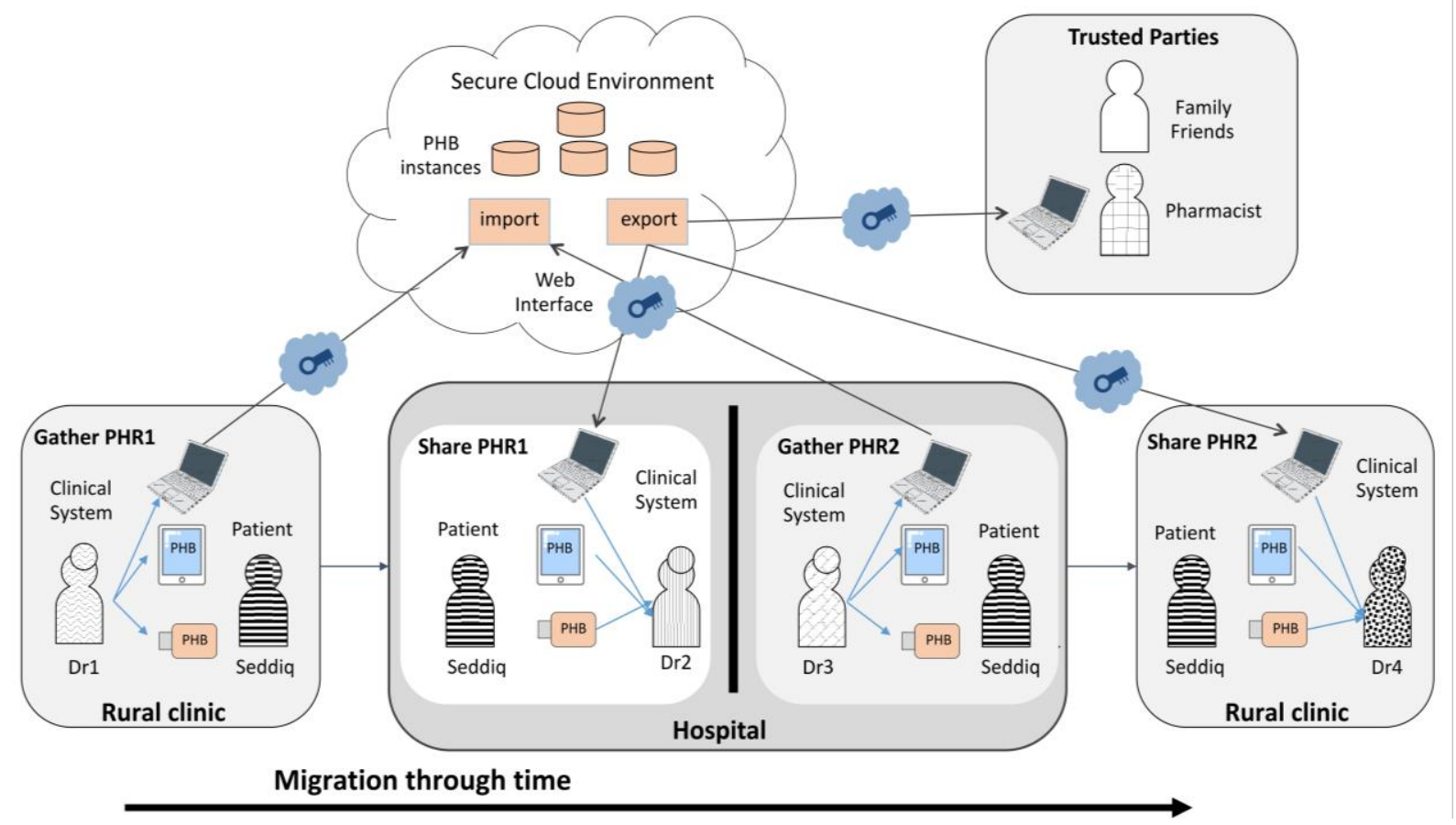

Fig. 1. The Portable Health Book use case

As low literate consumer in developing countries often migrate from rural villages to urban centers, the consumer should be able to ask another healthcare administration or doctor in a hospital to import their latest PHR or health history from the USB or Smart Card they are carrying. This will give the health clinic an improved perspective on the consumer's medical history. Following the consultancy, the patient can ask for the results to be imported to the USB or Smart Card. The PHRs will increment and the consumer will ask the next health clinic to view the appended PHRs and so on.

To provide robustness to the Personal Health Book application requires extending the storage options to the low literate consumer. The ability to save the PHR to an external storage such as a cloud environment will overcome the risk of physically losing the USB/Smart Card or file corruption. In this instance, the consumer will ask the healthcare provider or a family member to export the PHR to a secure website. The consumer will need a username and a password to access their unique remote $\mathrm{PHB}$ database.

A cloud-based PHB service has the benefits that the low literate consumer can use a smart phone PHB App to manage and store the PHR both on the smart phones and at the internet site. Once authorized by the consumer (health information owner), other interested parties such as family, friends and ultimately healthcare professionals, such as doctors, can view and import PHRs.

An example use-care for the PHB is shown in Fig. 1. Seddiq is a low literate middle-aged male consumer with hypertension and is examined at the rural clinic in Bangladesh. He would like to import the PHR. He has a choice of USB Smart Card with a PHB application (5 MB); a smart phone with PHB App but he needs a transmission method for importing the file; or asking the healthcare provider to export 
the PHR to a website (he has to type in the username and password). Seddiq, later migrates to urban city, overeats and feels unwell. He visits the hospital and allows the administration to access and import his PHB. He chooses the website method and shares his username and password. After the consultation, he asks the administration to export a copy of the PHR to the web-based PHB. Seddiq's family rely on his earnings, they are concerned his health status, and occasionally view a summary of his PHRs. During the festival season, Seddiq returns to the rural village and visits the health clinic for a check-up. Due to Internet outage, the community health clinic import his health records from his USB Smart Card. Using this approach, Seddiq, the people that depend on him, and health providers, have a convenient method to manage the PHR and make important decisions.

\section{B. Personal Health Book and Interoperability}

A unique characteristic of the PHB is that it tries to overcome interoperability issues by using a common file formats such as XML. This is out of synchronization with the current interoperability expectation to use HL7 as a common interface between healthcare providers. However, in developing countries, most health clinics have not implemented HL7 and have severe budget constraints. Thus in low resource areas, it is more pertinent to use the existing computers systems, databases and standard interfaces. Accepting this, there is still the problem that each health clinic may have its own data formats and structures. The PHB role is to accept the health clinic file, efficiently store the file, present the file to the PHB owner in a unified view, and create an understandable report to the next health clinic. To achieve this goal there are three main PHB functions:

Gather: PHRs are imported medical records from various medical sources (clinics, hospitals); PHRs contents are read; and stored it in the PHB database.

Store: PHRs will have different data characteristics: data type (integer, char, date); format (size of each data, storing order of data) and data structure (names and number of columns). PHB database will accommodate all incoming data in sufficient and reliable manner. The PHB owner should be able to understand the imported data.

Share: The PHB owner should be able to access, understand, manage and share the data with (clinics, doctors and family) and have full control of it.

\section{Privacy and Security}

In this paper we are introducing the primary concept for the Personal Health Book. Personal privacy and data security are under examination. PHB data will be securely stored into the PHB databse by encrypting all personal data [14], and no access will be granted to anyone without securely logging in the system [15] by having a user name and password [16]. The PHB can get higher security by incorporating the geomatric approach [17] or the token system [18] during login attempts.

\section{PHB TECHNICAL DESCRIPTION}

Personal Health Book is a software application for carrying a patient's health information as PHRs. The main criterion for the design is application size so it can run on any device including a smartcard chip. We focus on optimizing and simplifying the logic so the application can be implemented in budget-constrained environments.

\section{A. Importing from Source Health Clinic}

To import a PHR from a health clinic, the current concept focuses on XML file format and has basic expectations. To achieve a successful import to the PHB database, the following is required:

- File format: XML

- XML structure:

○ Group items by component types

- Each consecutive component must be nested to its parent

- For more than one word component name, a component must have "<type $>$ " item with the value of the component's name.

- Items (Fields Naming): Correct English spelling

- Minimum component types:

$$
\begin{array}{cl}
\circ & \text { Patient } \\
\circ & \text { Hospital/Clinic } \\
\circ & \text { Physician/Doctor } \\
\circ & \text { Nursing/Checkup }
\end{array}
$$

These set of rules are translated into an XML schema file (XSD) used for XML validations, which also helps in the logic of reading the XML file content.

\section{B. PHB Import Logic}

To import the XML file from the source health clinic to the PHB database (see Fig. 2), we designed an algorithm to import PHRs coming from different sources. The steps are as follows:

1) Check imported file extension:

- If it was not an XML, go to step 4.

2) Validate $X M L$ file structure against a predefined $P H B$ schema (XSD):

○ If it was not valid, go to step 4.

3) Open XML file and read its contents:

a) Get all parent nodes

b) For each parent node, check it's availability in the "Categories" table in the PHB database:

- If it does not exist, create a new category 


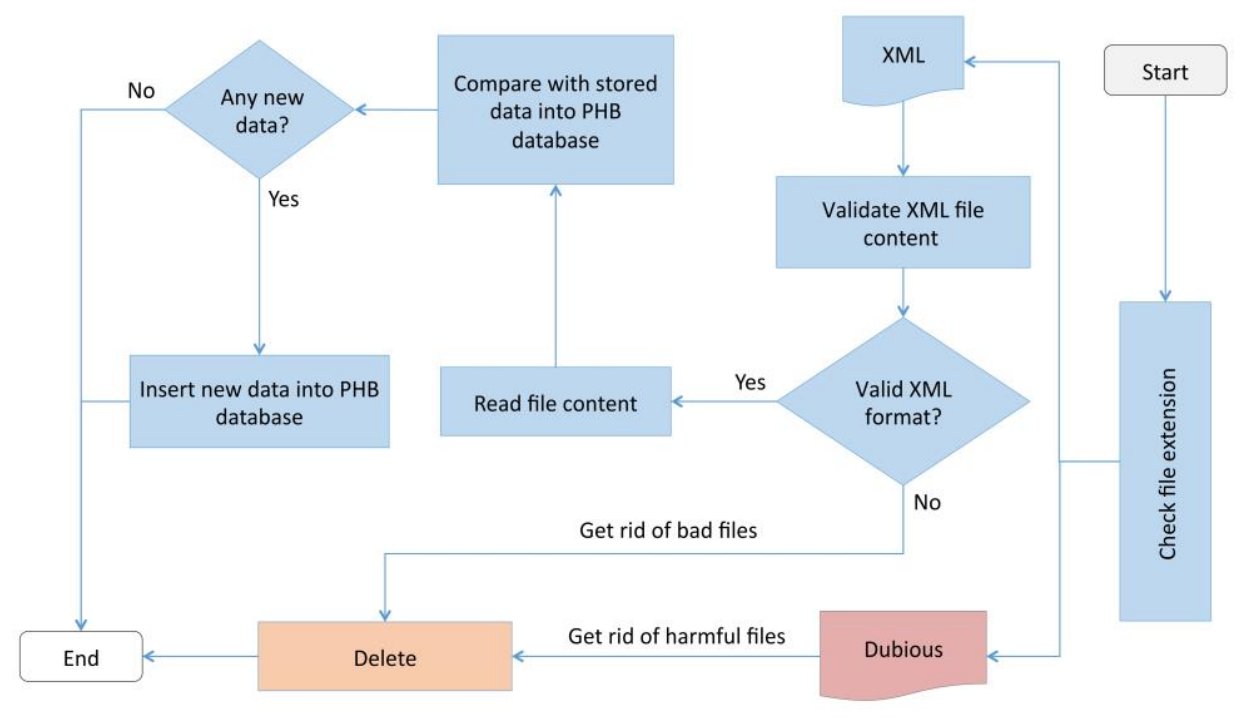

Fig. 2. The PHB Workflow for Gathering and Storing PHRs

4) Close the XML file

c) or each child under each parent, check it's availability in the "Items" table in the PHB database:

○ If it does not exist, create a new item

d) For the existing category, check if imported child node match the number and order under existing templates in the PHB database

e) Create new templates for new categories and for the mismatch cases from point $d$ ).

f) Insert new values into the "Values" table in the PHB database according to templates ordering of the items

g) Insert new records for new categories and link them to their values and templates

h) Insert related medical records and link them to their sources, values and templates

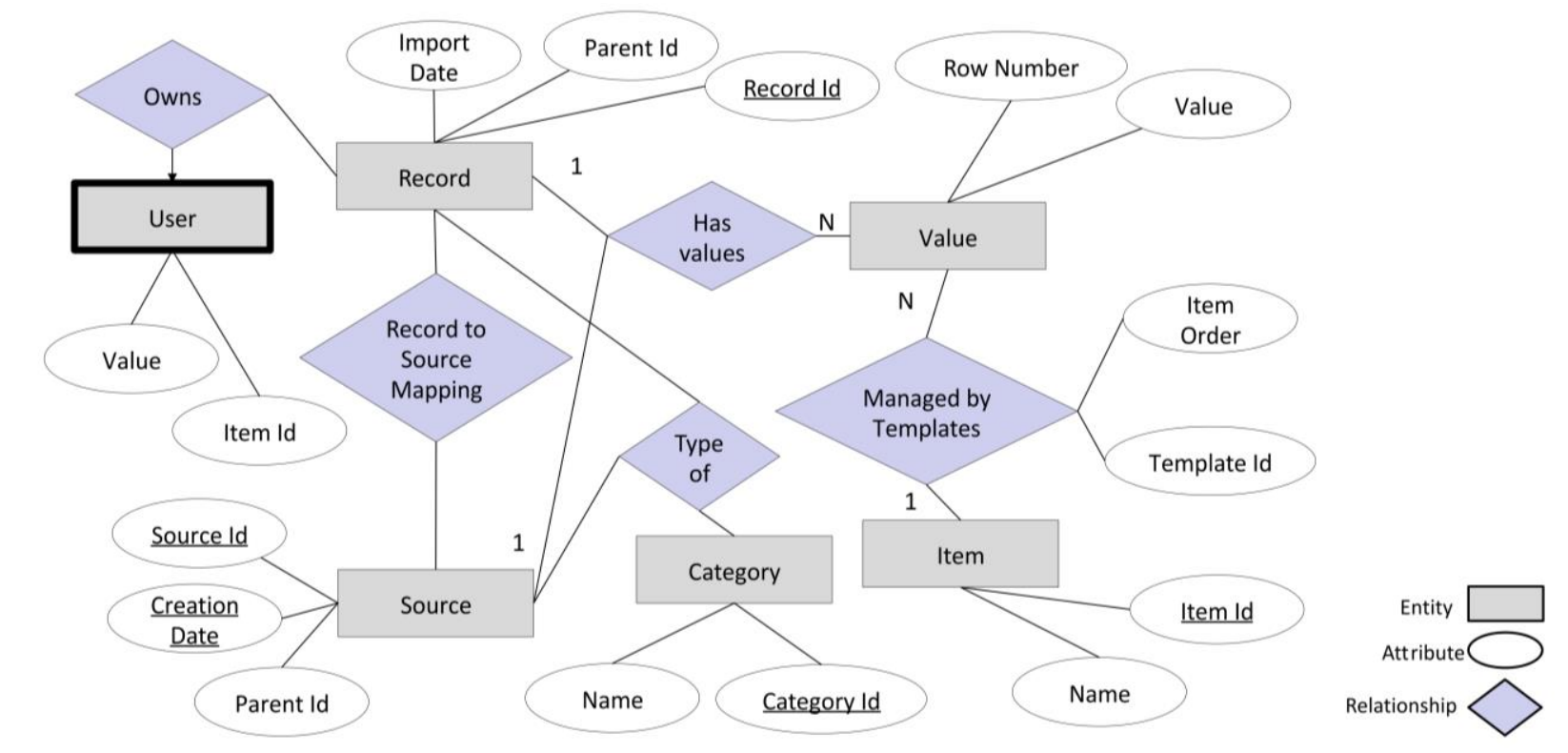

Fig. 3. Entity Relationship Model for the PHB Database

\section{PHB Database}

The PHB database needs to manage files and data from multiple healthcare sources; it should adapt any new data type and grow dynamically. It should work with future changes in the data without the need to change the database structure.

The entity relationship data model for an adaptive PHB database is shown in Fig. 3. The ER diagram depicts the interconnections and relationships between entities: User, Record, Source, Category, Value and Item. For example, User and Record are connected via the relationship "Owns" (one-tomany). In other words, the user will own many records. Similarly, entities Source and Record are connected via the relationship "Imported from". Here, a record will be imported from a source. In another scenario, Record and Value are connected via "Has values". Which means, a record will have 
many values. Similarly, other connected entities have relationships in a meaningful way.

a) PHB database design: The PHB database consists of 6 tables. We used MS SQL to create basic database concepts that will work with all database engines. The tables and their functions are described as follows:

- User Profile table: User personal details, such as name, date of birth, and address.

- Items table: All data naming/labels are stored as an index (including unknown new items). An item can be name, age, temperature, height and description.

- Categories table: This table is used to manage data types, such as hospital, doctor, drug and checkup details.

- Templates table: As PHB accepts all data coming from different sources with different structures, there is a need to keep track what items related to each source.

- Records table: All health records are stored in records table as well as related records. For example, one hospital visit record has prescribed drugs and lab reports; these records have their own related data, different elements and more than one occurrence. They will be saved as sub-records in the records table. Even records source details will be kept in this table, such as hospitals, doctors, and drugs. A source can be sub of another source (doctor is sub of hospital). Each record sored in the database, will be related to a certain source, such as, a doctor can be a source of a record, or a drug is the source of the prescription. All sources (Hospital) to sub-sources (Doctor) and records (Checkup details) to sub-records (Prescriptions) are linked through "ParentId" column in the "Records" table.

- Values table: All the values of the items that are specified in the templates table are stored here, which is actually the values of the sources and records details.

The database is decomposed and designed in such a way that there will never be a "Null" value or a blank stored in any table (Fig. 4).

b) Querying the PHB database: Every source and record will have a set of values linked together by the template table. To query the PHB database, the templates table will identify the items (naming/labeling) related to each value of the queried data type (source/record). Items related to the values are stored in the Items table, and every template is linked to a number of these items. If a hospital changes the number of items, it will not affect the existing stored data because the date instance is kept. An example of a query would be to type in a keyword such as 'Blood Pressure' between selected dates and the PHB would present the report.
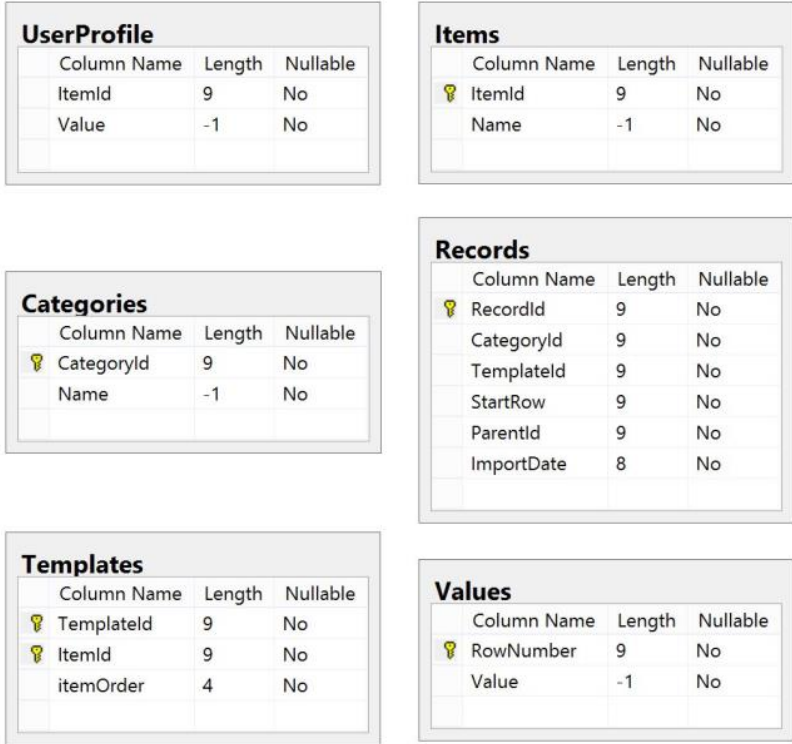

Fig. 4. The PHB Decomposed Database Design

\section{Share Stored PHRs with a Doctor from Another Clinic}

The PHB has the ability to share the patient's PHRs visually through PHB application directly, so a doctor can understand the patient's health history, in addition a report can be generated and exported to another system.

To export a PHR report from the PHB database to a destination (seeking) health clinic, the current concept will generate an XML. To achieve a successful export that can be understood by the seeker, the following steps will occur:

1) Get all root sources

2) Create new blank XML file and open it to be filled

3) For each root source, get its details (by referring to templates)

a) Get all child sources of the root source

b) For each child source, get its details (by referring to templates)

c) Get all parent records of each child source, get its details (by referring to templates)

d) For each parent record get all sub-records that their sources are sub-sources of the child source, get its details (by referring to templates)

e) Get sub-records that their sources are not sub-source of the root source, get its details (by referring to templates)

f) Fill into the XML file all collected data

4) Close the XML file and publish it

\section{USER INTERFACES}

This section indicates how a consumer can use the PHB when ported onto a USB card. Whenever the patient visits a hospital, the following steps occur: 


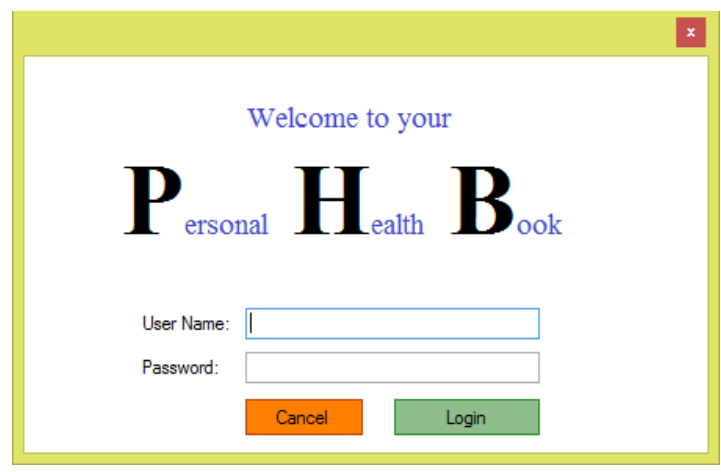

Fig. 5. Log in screen

1) The pateint gives the USB smart card to the doctor or health-worker to plug it in their personal computer.

2) The doctor/health-worker runs the PHB application, and a login screen will appear.

3) The patient has to insert his username and password as shown in Fig. 5.

4) After a successful login, the PHB's main window will appear, and the user can navigate through the application via the main menu (see Fig. 6).

The menue has three main items:

- Reports: Where patient can view and share his health related reports (Health Reports) or understand his data statiscally (Statistical Reports)

- Import PHR: Where doctor/healthcare worker can import new PHRs

- Logout: It is important that the patient logs out after he finishes from the PHB application to keep his data securely safe.

5) If (Health Reports) was selected, health reports form will appear (see Fig. 7), here the patient can view his health related data.

6) The (Generate Report) button will produce the health report based on the patient's customisation and filtering.

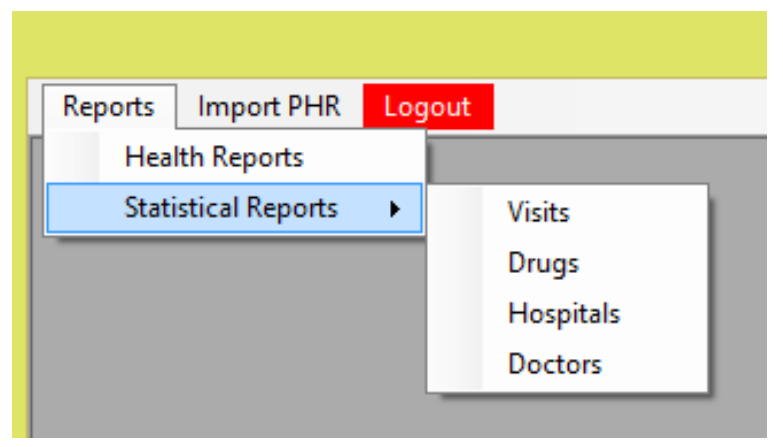

Fig. 6. The PHB Application Main Menu

7) By clicking on the (Export) button, the patient can share a copy of the produced report with the doctor/healthworker, either in an XML or PDF format. As a secuerity and privacy protection measure, an authenticaion form will appear and the patient will have to enter his credintials to allow the export process.

8) After the patient finishes the health checkup, the doctor/health-worker will be able to import the new PHR to the PHB application by selecting (Import PHR) from the main mеnu.

9) To quit the application, the patient simply clicks on (Logout).

\section{TESTING THE PHB}

\section{A. Importing real PHRs and viewing the output}

To test the PHB framework we randomly selected a real patient PHR (with personal details deleted) from the Grameen Portable Health Clinic database in Bangladesh (22,000 entries). We accessed 2 PHR records from a Japanese hospital "Hospital xyz" (representing an urban city) issued at different times. We imported 3 PHRs into the PHB environment. The PHB role was to reorganize the data from two health clinic visits by categorizing and grouping and presenting a unified, human understandable, view structure.

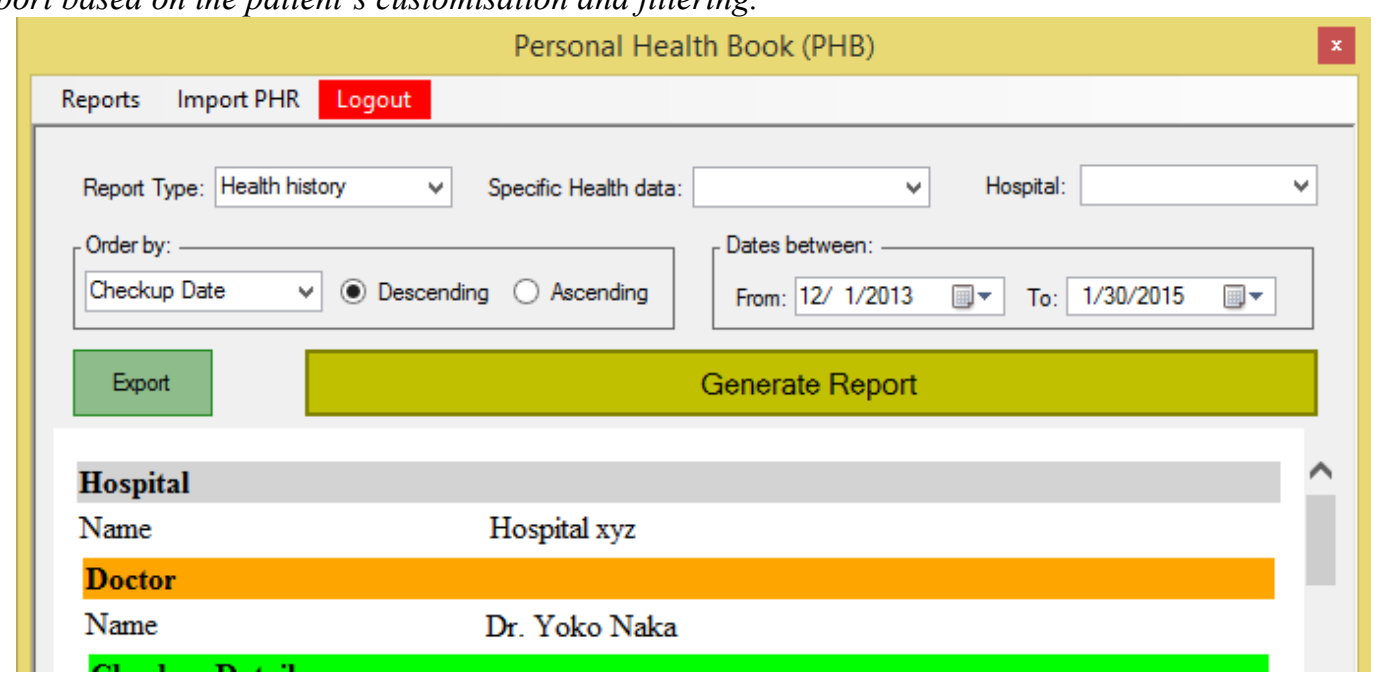

Fig. 7. Health Reports form 
The Portable Health Clinic normally generates PDF files to print PHRs. For the test, we created an XML file to match the import requirements (see Fig. 8). The data was successfully imported in the PHB database.

We implemented an experimental version of the PHB (USB card version) to test the readability and the capability of the PHB application. In which the consumer can export generated report as a PDF of XML file to share it with his doctor, family or hospital. Because the PHB database is designed to get the use of each entity stored, it can produce health related reports as well as statistical reports such as number of visits, drugs, hospitals and doctors. These types of reports can help in understanding the extent a person is following up on his health and the address and contacts for all visited hospitals and doctors.

The PHB patient report is human readable, understandable and meaningful as seen in Fig. 9. The reports of multiple visits from two medical firms are shown in a descending order. Every category/type in the report is clearly separated from the others and contains the exact number of items that the original record contained. In addition, all child's components are displayed under their parent source.

\section{B. Testing the impact of many health clinic visits}

To assess the impact of visiting many health clinics and subsequent creation of PHRs on the PHB application data size, we prepared a simulation to observe the rate of size growth in bytes every time a health record is imported (see Fig. 10). This is important because we do not want the PHB application to grow and exceed the memory size of the USB card. The simulation involved a patient visiting 2 hospitals a total of 24

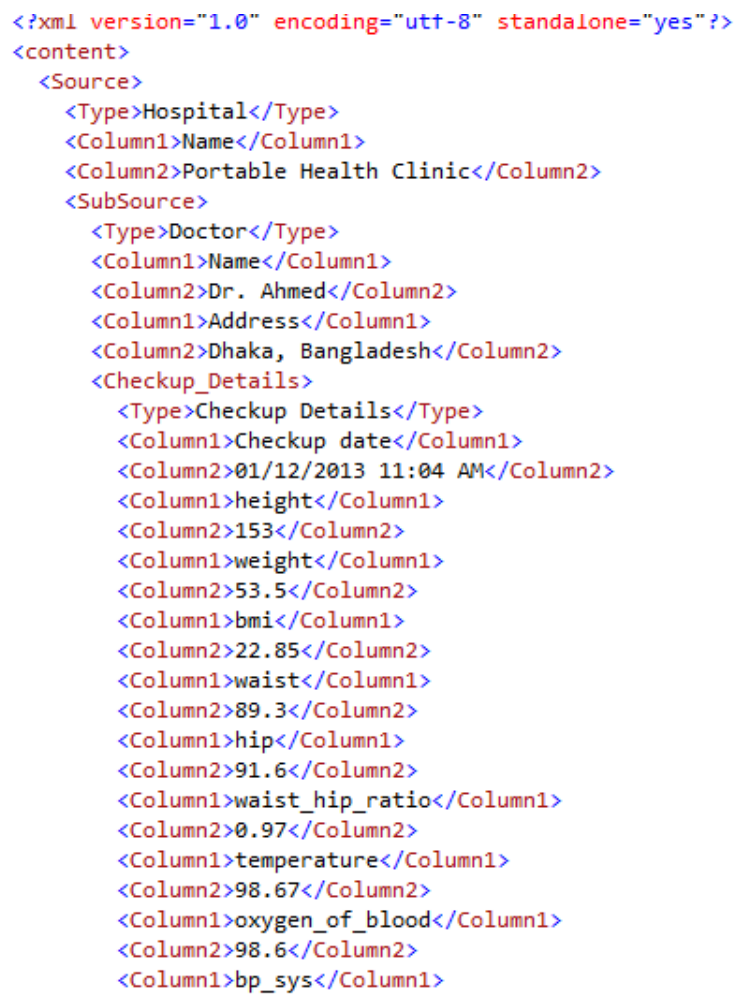

Fig. 8. Imported EHR from PHC database, Bangladesh times and generating 24 PHRs. The name of doctor was randomly changed and whether drugs were prescribed or not was randomly selected. The simulation output showed that the first imported PHR into the system consumed a large memory

\begin{tabular}{|c|c|}
\hline Hospital & Last Visit \\
\hline Name & Hospital xyz \\
\hline \multicolumn{2}{|l|}{ Doctor } \\
\hline Name & Dr. Yoko Naka \\
\hline \multicolumn{2}{|l|}{ Checkup Details } \\
\hline Checkup date & $24 / 01 / 201510: 00 \mathrm{AM}$ \\
\hline Metabolic & No \\
\hline Medical History & No \\
\hline Advice on Treatment & No \\
\hline Blood Pressure & $84 / 52$ \\
\hline Hospital & Older Visit \\
\hline Name & Portable Health Clinic \\
\hline \multicolumn{2}{|l|}{ Doctor } \\
\hline Name & Dr. Ahmad \\
\hline Address & Dhaka, Bangladesh \\
\hline \multicolumn{2}{|l|}{ Checkup Details } \\
\hline $\begin{array}{l}\text { Checkup date } \\
\text { weight }\end{array}$ & $\begin{array}{l}\text { 08/08/2014 10:00 AM } \\
53.5\end{array}$ \\
\hline BMI & 22.85 \\
\hline waist & 89.3 \\
\hline hip & 91.6 \\
\hline waist_hip_ratio & 0.97 \\
\hline
\end{tabular}

\begin{tabular}{|c|c|}
\hline Hospital & Older Visit \\
\hline Name & Hospital xyz \\
\hline \multicolumn{2}{|l|}{ Doctor } \\
\hline Name & Dr. sanji Jiro \\
\hline \multicolumn{2}{|c|}{ Checkup Details } \\
\hline Checkup date & $21 / 06 / 2014$ 10:30 AM \\
\hline Chest & 84.0 \\
\hline BMI & 23.1 \\
\hline Fat level & $+5.0 \%$ \\
\hline Blood Pressure & $102 / 60$ \\
\hline Doctor & Oldest Visit \\
\hline Name & Dr. Yoko Naka \\
\hline \multicolumn{2}{|c|}{ Checkup Details } \\
\hline Checkup date & $30 / 12 / 201311: 04 \mathrm{AM}$ \\
\hline Blood Pressure & $120 / 72$ \\
\hline \multicolumn{2}{|c|}{ Body Measurements } \\
\hline Height & 167.4 \\
\hline
\end{tabular}

Fig. 9. PHB Personal History Report 


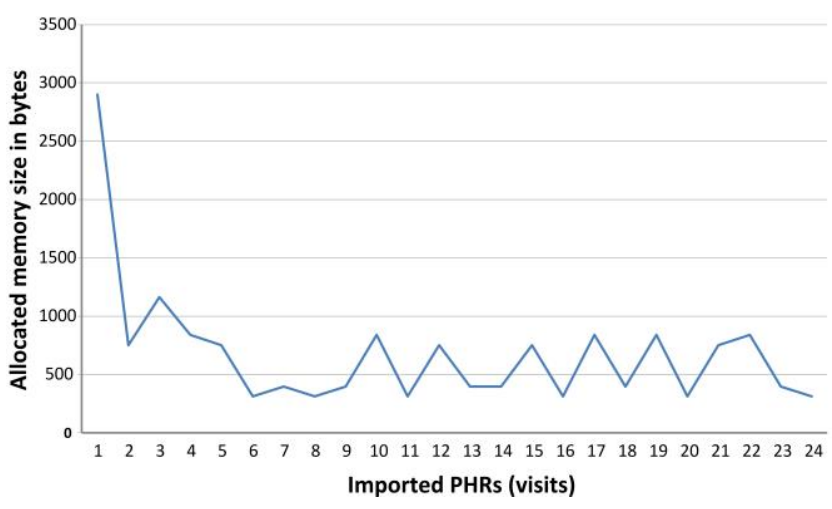

Fig. 10. Database Size Increment vs. Number of Imported Records

size as new items, categories and templates were created by the PHB database. The following imported PHRs consumed much less memory size as the database structure had been set up. When new items/categories are created they consume memory but less than the initial set up. Therefore, Fig. 10 shows a drop in bytes after the first PHR and then moves to a steady state as a few new items/categories are introduced. When the consumer visits the same hospital with same doctor and receives the same medication there will be minimal impact to PHB memory consumption (PHR number 7, 9, 13, 14 and 23).

\section{RESULTS \& DISCUSSION}

The PHB test results demonstrate that PHR XML files can be imported, the patients' characteristics and medical data stored in a decomposed database and human readable reports can be generated. A subsequent XML file imported to the PHB, such as the next medical check-up in a different source health clinic, will append the data and appear above the existing data. A healthcare worker at another health clinic reading the report can export a PDF or XML copy to their system or print the report and manually update their system. Following the health consultancy, the patient will ask the healthcare worker at the clinic for a digital report containing the results to be imported into the PHB.

The impact of visiting different health clinics over time on the PHB memory requirements is minimum. The decomposed database can grow dynamically as new data types are added (the customer has a new disease or health problem) and is designed that there will be no null or blank values stored in a table leading to optimized memory management. The simulation indicated an average of 500 bytes per PHR per visit. With this indication, we can assume that an average patient can store a lifetime of personal health history on a USB Card.

There are many challenges for the widespread adoption of a PHB in developing countries. Health clinics in the rural areas may not have IT systems and cannot give digital records (XML files) to their patients. The source health clinic may not want to share the patient's health records with the patient. The seeking health clinic may not want to accept an unknown patient's PHB in fear of a computer virus. There may be affordability and literacy issues with USB sticks or accessing the internet. There are security and privacy concerns and many technical questions. However, a PHB implementation would introduce a robust patient-centred healthcare model and a unique opportunity for low-income individuals to manage their own health in many countries where healthcare systems are under severe pressure.

\section{CONCLUSION}

Personal Health Book software is an application that manages Personal Health Records and is suitable to developing countries where low-literate consumers migrate from rural areas to urban areas and who want to know their medical history. It provides free aid to low resourced healthcare institutions facing difficulties in implementing Electronic Health Systems by allowing the institutions to save standard files in their existing databases. By porting the Personal Health Book application onto devices such as USB, smartcard or lowcost smartphone with connection to cloud computing environment, will enable low literate consumers to access their own medical records even if they migrate to another country.

The PHB application is a single-user database smart health convenience option for the consumers rather than a medical necessary. A PHB cloud-based solution is an aggregate of many single personal unique databases rather than a large common database. There are many risks to storing all PHRs in one location, such as privacy, theft, and non-acceptance by health professionals and this is beginning of the list. However, like most tools of convenience it will take time and modification before becoming generally adopted. The most important PHB attribute is that allows individuals to easily store, access and review their own personal digitized health history. The PHB is thus more relevant to the individual than to the institution that may use it to make clinical decisions. The PHB is also relevant for individuals who are increasingly becoming interested in quantifying their lifestyles and are using sensors to measure and make sense of their wellness over time.

\section{FUTURE WORKS}

Personal Health Book application requires more development and testing. The application requires robust security measures to protect the consumer's private health data using a strong authentication and encryption method and against unwanted external intrusions. Error and correction management to handle file corruption during PHR import process. The cloud environment architecture will be defined. Testing involves importing different types of PHRs from health clinics and their departments. Checking if health clinics can import a real PHR stored on the PHB to their data system and researching the associated problems. Finally, surveying endusers - low literate consumers and medical professionals - on the applicability of the PHB to their lives.

\section{ACKNOWLEDGMENT}

We thank Global Communication Center, Bangladesh, for access to and use of personal health records from Portable Health Clinic database.

\section{REFERENCES}

[1] Claude Sicotte, Jean Louis Denis, Pascale Lehoux and Francois Champagne, "The computer based patient record challenges towards timeless and spaceless medical practice”, Journal of Medical Systems, vol 22, no.4, pp.237-256, 1998. 
[2] Marion J. Ball, Melinda Y. Costin and Christoph Lehman, "The personal health record: Consumers banking in their health", Stud. Health Technol. Informat., vol 134, pp.35-46, 2008.

[3] Robert Steinbrook, "Health care and Amercian Recovery and Reinvestment Act" N.Engl. J.Med., vol 360, no 11, pp.1057-1060, Mar 12, 2009

[4] Maisie Wang, Christopher Lau, Fredrick A. Matsen and Yonfmin Kim, "Personal Health Information Management System and its Application in Referral Management", IEEE Trans on Information Technology in Biomedicine Vol.8., No. 3. September 2004.

[5] Healthcare Information Management and Systems Society (HIMSS). (2007). HIMSS personal health records definition and position statement. Retrieved October, 22, 2009, from http://www.himss.org/content/files/phrdefinition071707.pdf

[6] Kim Kyungsook and Eun-shim Nahm, "Benefits of and barriers to the user of personal health records (PHR) for health management among adults", Online Journal of Nursing Informatics (OJNI), 16 (3). http://ojni.org/issues/?p=1995

[7] Kim Nazi, "The Personal Health Record Paradox: Health Care Professionals' Perspectives and the Information Ecology of Personal Health Record Systems in Organizational and Clinical Settings", Journal of Medical Internet Research. Vol 15 No 4. April 2013. http://www.jmir.org/2013/4/e70/

[8] Juha Puustjarvvi and Leena Puustjarvi, "Personal Health Book: A Novel Tool for Patient Centered Healthcare", In the Proc of the International Conferenece on Health Informatics (HEALTHINF 2011).pp 386-393, 2011

[9] Juha Puustjarvi and Leena Puustjarvi "Personal Health Book: A CloudBased Tool for Patient Centered Healthcare", Journal of Public Health Frontier. Vol 2., Iss 3., pp 146-155, Sept 2013.
[10] Seddiq Alabbasi, Andrew Rebeiro-Hargrave, Ashir Ahmed, Kazuaki Murakami, and Hirohito Yasuura, "Personal lifetime data and its smart management" Proceedings of the 2013 International Symposium on Intelligent System Engineering (ISISE 2013), November 15-16, Abu Dhabi, UAE

[11] Weihua Chen and Metin Akay, "Developing EMRs in Developing Countries", IEEE Transactions of Informationa technology in Biomedicine, Vol 15, No. 1 January 2011

[12] Francine L. Maloney and Adam Wright, "USB-based personal health records: An analysis of features and functionality", Int. J. Med Informat", vol 78, no.2, pp97-111, Feb 2010

[13] A. Ahmed, A. Rebeiro-Hargrave, N. Yohara, E. Kai, R. Hossein, N. Nakashima "Targeting Morbidity in Unreached Communities Using Portable Health Clinic System". IEICE Transactions on Communications, Vol.E97-B, No.3. March 2014

[14] Rajitha Tennekoona, Janaka Wijekoona, Erwin Harahapa, Hiroaki Nishib, "Per-hop Data Encryption Protocol for Transmitting Data Securely Over Public Networks", Procedia Computer Science, Vol 32, pp. 965-972, 2014

[15] Stuart P. Goringa, Joseph R. Rabaiottib, Antonia J. Jonesb, "Antikeylogging measures for secure Internet login: An example of the law of unintended consequences", Computers \& Security Vol 26, Issue 6, pp. 421-426, September 2007

[16] Khosrow Dehnad, "A simple way of improving the login security", Coputers \& Secruity vol 8, Issue 7, pp. 607-611, Novemeber 1989

[17] Tzong-Chen $\mathrm{Wu}$, "Remote login authentication scheme based on a geometric approach", Computer Communications Vol 18, Issue 12, pp. 959-963, December 1995

[18] Yen Sung-Ming, Liao Kuo-Hong, "Shared authentication token secure against replay and weak key attacks", Information Processing Letters, Vol 62, Issue 2, pp. 77-80, 28 April 1997 